\section{PNEUMATOSE INTESTINAL}

\author{
PNEUMATOSIS INTESTINALES
}

\section{INTRODUÇÃO}

Pneumatose Intestinal (PI) é a presença de gás na parede do trato gastrointestinal (TGI). Constitui sinal clínico-radiológico que pode ocorrer em doenças diversas, e com significado igualmente variável. É provável que sua prevalência tenha aumentado nos últimos anos. A natureza das doenças subjacentes tem modificado nos últimos decênios. No passado, as úlceras pépticas eram a principal causa. As condições clínicas que cursam com estados de imunossupressão e aumento da permeabilidade da mucosa do TGI (AIDS, pacientes transplantados, em quimioterapia etc.) são, provavelmente, as atuais causas mais freqüentes de PI.

Este relato descreve um caso de estenose péptica do piloro, com detecção peroperatória de PI. Esse achado não interferiu na condução do caso e no prognóstico do paciente. $\mathrm{O}$ reconhecimento da PI foi fundamental para evitar que medidas inadequadas fossem tomadas.

\section{RELATO DE CASO}

Paciente do sexo masculino, 30 anos de idade, portador de úlcera péptica duodenal em tratamento clínico há nove anos, evoluindo, durante este período, com várias reinfecções pelo H. pylori. Apresentou, nos últimos dois anos, sintomas dispépticos dos tipos obstrutivo e refluxo; empachamento, distensão epigástrica, náuseas e vômitos pós-prandiais, pirose, regurgitação e perda ponderal de $4 \mathrm{~kg}$. A endoscopia digestiva alta e a radiografia contrastada do trato digestivo proximal evidenciaram estenose mesobulbar subtotal e esofagite de refluxo grave (Savary-Miller grau III). A dosagem da gastrina sérica foi normal.

\section{Luiz Fernando Veloso ${ }^{1}$ Marco Antônio Gonçalves Rodrigues, TCBC-MG²}

À laparotomia, diagnosticou-se estenose pilórica cicatricial, de $1 \mathrm{~cm}$ de extensão e $1 \mathrm{~cm}$ de diâmetro externo (Figura 1), e diversas lesões bolhosas de diâmetros variados na parede do íleo, em uma extensão de $60 \mathrm{~cm}$, localizadas em sua borda mesentérica e sem comunicação com a luz do TGI (Figura 2). Realizou-se vagotomia seletiva, antrectomia e gastroduodenostomia na pequena curvatura, e biópsia das lesões bolhosas. Detectou-se pneumoperitônio que, apesar de mais volumoso que o usualmente observado, regrediu espontaneamente a partir do quarto dia de pósoperatório (DPO). O paciente evoluiu satisfatoriamente e recebeu alta no sétimo DPO. Ocorreram alívio completo dos sintomas e ganho de peso. O exame anatomopatológico das biópsias das lesões bolhosas mostraram-se compatíveis com pneumatose intestinal.

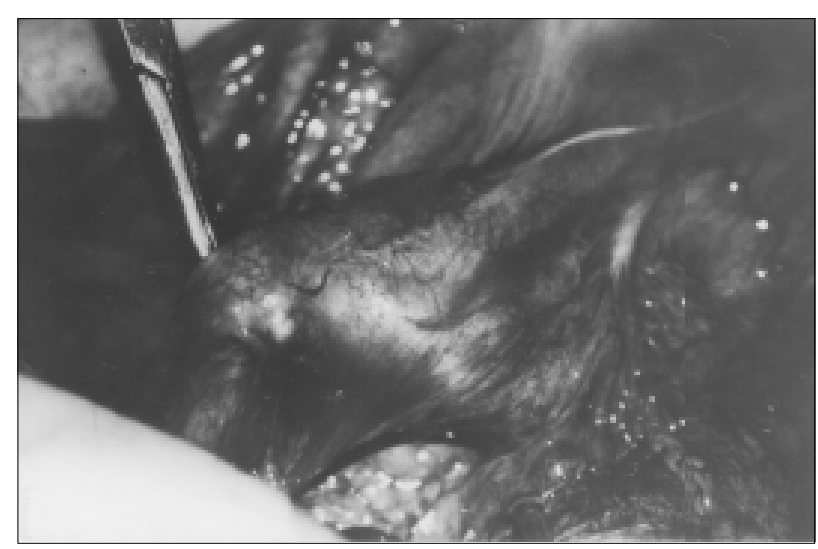

Figura 1 - Estenose pilórica. Aspecto peroperatório. No "passafio", piloro estenosado em uma extensão de $1 \mathrm{~cm}$ e com aproximadamente $1 \mathrm{~cm}$ de diâmetro externo. Observa-se ainda estômago dilatado.

1. Residente em Cirurgia Geral do Hospital das Clínicas da Universidade Federal de Minas Gerais.

2. Professor Assistente-Doutor do Departamento de Cirurgia da Faculdade de Medicina da Universidade Federal de Minas Gerais. Coordenador do Grupo de Esôfago, Estômago e Intestino Delgado do Serviço de Gastroenterologia, Nutrição e Cirurgia do Aparelho Digestivo (GEN-CAD) do Hospital das Clínicas da Universidade Federal de Minas Gerais.

Recebido em 10/08/2000

Aceito para publicação em 08/02/2001

Trabalho realizado no Serviço de Gastroenterologia, Nutrição e Cirurgia do Aparelho Digestivo do Hospital das Clínicas da Universidade Federal de Minas Gerais. 


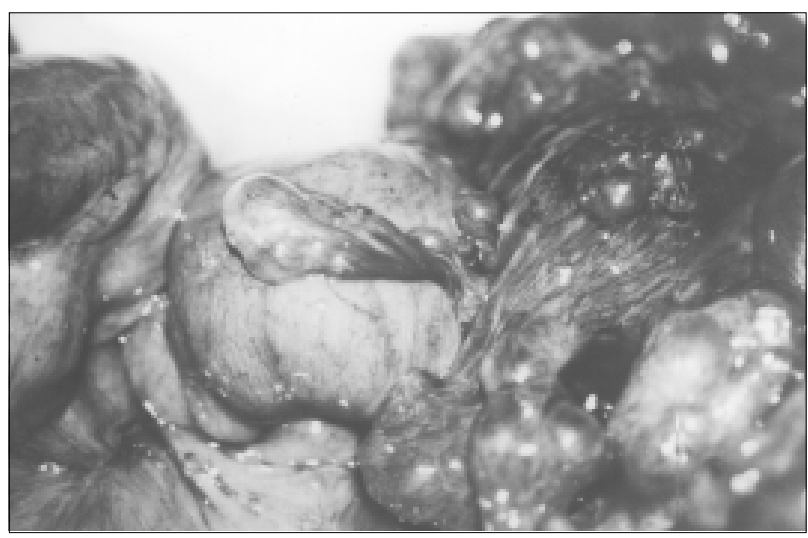

Figura 2 - Pneumatose intestinal. Aspecto peroperatório. Diversas lesões bolhosas de diâmetros variados na parede do íleo, em uma extensão de $60 \mathrm{~cm}$, localizadas em sua borda mesentérica e sem comunicação com a sua luz.

\section{DISCUSSÃO}

O mecanismo pelo qual uma coleção de ar, quase sempre estéril, se forma na parede de um segmento do TGI é controverso. Segundo as teorias mais aceitas, o ar poderia alcançar a parede do intestino a partir dos alvéolos pulmonares ou da luz do TGI.

Após ruptura das paredes dos alvéolos, $\mathrm{o}$ ar dissecaria ao longo dos brônquios centripetamente em direção ao mediastino, e através do retroperitônio, ao longo dos vasos do abdome, alcançaria o mesentério e a parede do intestino. As doenças pulmonares obstrutivas crônicas (DPOC) e o trauma contuso do tórax seriam causas de PI por este mecanismo.

O ar que ocupa a luz do TGI poderia alcançar uma das camadas da parede do intestino através de dois mecanismos: por perda da continuidade da mucosa ou por aumento de sua permeabilidade. São muitas as situações que cursam com perda de sua continuidade: úlcera péptica, isquemia, inflamação e infecção da parede intestinal. Condições clínicas e afecções que evoluem com imunossupressão poderiam causar PI por aumentar a permeabilidade da mucosa. Disfunção da "barreira mucosa" do intestino permitiria a passagem de ar, como ocorre com as bactérias e os metabólitos ${ }^{1,2}$.

Na revisão realizada por Koss ${ }^{3}$, dos 213 casos de PI diagnosticados, $55 \%$ foram atribuídos a úlceras pépticas gástricas ou duodenais. Revisões que se seguiram relataram, com frequiência, associação com DPOC e isquemia mesentérica ${ }^{1,4}$. Um número progressivamente maior de relatos de casos relacionados a estados de imunossupressão, neoplasias (especialmente as hematológicas), doenças inflamatórias intestinais e do tecido conectivo, tem sido registrado nos últimos anos ${ }^{1,2,4}$. Atualmente estas têm sido as principais causas de PI.

Para conduzir racionalmente um paciente com PI, além do diagnóstico do achado, é fundamental que se reconheça sua causa e seu significado.
A PI idiopática, presente em $15 \%$ a $30 \%$ dos casos, em geral, não apresenta significado clínico e não exige medidas terapêuticas específicas. Nestes casos, basta reconhecer a PI para que nenhuma medida indevida ou precipitada seja tomada.

Em outras situações, a PI poderá ser diagnosticada até mesmo antes que sua causa seja encontrada. As afecções que, mais freqüentemente, apresentam-se desta forma são: úlceras pépticas, estenoses do piloro, diverticulite, doença celíaca, Hirschsprung, doença de Crohn, pós-operatórios de operações com abertura da luz do TGI, pósendoscopias (diagnósticas ou terapêuticas) e pós-enemas baritados, ingestão de substâncias corrosivas, doenças pulmonares crônicas, trauma torácico contuso e doenças do colágeno. Quase sempre, a PI não tem implicação prognóstica nestes casos. A esclerodermia e a esclerose sistêmica progressiva constituem exceções a esta regra, para as quais a PI constitui sinal de estádio avançado da doença.

No presente caso, a PI foi um achado ocasional após o diagnóstico de sua causa, e não interferiu no tratamento e no prognóstico do paciente.

A PI pode também estar associada a estados clínicos que exigem tratamento de emergência. São exemplos: isquemia mesentérica (onde, quase sempre, a PI associa-se a necrose intestinal), a gastrite enfisematosa e as perfurações do tubo digestivo. Na enterocolite necrotizante é, usualmente, sinal de diagnóstico tardio e prognóstico sombrio. A necrose intestinal constitui a condição mais grave que se associa à PI e precisa ser sempre lembrada ${ }^{1}$. A interpretação do significado da PI deve ser mais cuidadosa nos casos de obstrução intestinal, retocolite ulcerativa, colites neutropênicas e intussuscepção. Nesses, a PI pode ou não significar sofrimento isquêmico de alças intestinais.

Na AIDS, a PI está associada, freqüentemente, à criptosporidiose ${ }^{5}$, embora já tenha sido encontrada em associação com infecções por rotavírus, cytomegalovirus, pseudomonas e clostridiuns. Representa sinal de doença avançada. Em pacientes transplantados ou em quimioterapia, sua presença coincide, quase sempre, com períodos de neutropenia.

Alguns achados clínicos podem contribuir para a interpretação do significado da PI e para a definição da conduta. Quando ocorre em recém-nascidos, devemos considerar o diagnóstico de enterocolite necrotizante $(\mathrm{EN})^{2}$. Ela é mais provável em recém-natos com menos que dois meses de idade, em associação com enterorragia e distensão abdominal e com evidência, nos exames de imagem, de gás na veia porta ${ }^{2}$. Se a PI é o único achado em crianças com idade acima de dois meses, é conveniente mais cautela antes indicar a laparotomia. Neles, a PI pode ocorrer em associação a outras condições como a síndrome do intestino curto e a fibrose cística ${ }^{2}$.

Independentemente da faixa etária, a laparotomia será, provavelmente, benéfica quando a PI estiver presente nos seguintes casos: sangramento retal, febre, obstrução intestinal, irritação peritoneal, leucocitose, acidose metabólica ou láctica, sinal radiológico de gás na veia porta ou em vasos mesentéricos. 
O pneumoperitônio está presente em aproximadamente $30 \%$ dos pacientes, mas apenas um quarto deles necessitarão serem operados. Este fato representa um desafio para o cirurgião.

Muitos pacientes que apresentam PI usam corticosteróides, imunossupressores ou são portadores de doenças que induzem imunossupressão. Em decorrência deste fato, eles podem apresentar exame clínico pouco alterado, mesmo que tenham doenças que demandem laparotomia de urgência.

Os métodos de imagem podem, freqüentemente, identificar padrão de radioluscência ramificado em posição intramural, gás em ramos terminais dos vasos mesentéricos ou na veia porta, quando houver isquemia, necrose ou infecção da parede do intestino. Contudo, este achado pode ser encontrado também em condições clínicas menos graves $^{1,2,4}$.

O risco de evolução desfavorável é menor quando o cólon é o único sítio anatômico acometido pela PI do que quando exclusivamente o delgado é acometido $(\mathrm{OR}=2,2)$ ou se ambos forem acometidos $(\mathrm{OR}=5,3)^{2}$. Isto justifica-se pelo fato da PI idiopática, em adultos, acometer, com maior freqüência, o cólon (principalmente o esquerdo) ${ }^{2}$. A PI pode se apresentar com distribuição linear, o que tende a ocorrer em condições mais graves quando comparada à forma bolhosa ${ }^{1}$.

Esse caso apresentado ressalta a importância do reconhecimento da PI e o valor da interpretação correta de seu significado clínico, para que a conduta seja estabelecida de forma racional e correta.

\begin{abstract}
Pneumatosis Intestinalis (PI) is the presence of gas-filled cysts within the wall of the gastrointestinal tract (GIT). It is a clinical and/or radiological sign associated with a wide spectrum of diseases, so that it has a variable clinical significance. Probably, its prevalence is increasing. The nature of the diseases causing PI is been modifying in last decades. Peptic ulcers were its main cause in the past. Nowadays, probably, immunosuppressive conditions and states of increased permeability of the GIT mucosa (AIDS, transplanted patients or in chemotherapy, etc) are more common causes. PI can be shown on simple abdominal roentgenograms and computed tomographic scans obtained with lung windows. Its diagnosis include definition of the cause in addition to its presence. The treatment should be directed to the cause of the PI, fluctuating from expectant to emergency laparotomy. The present report is a contribution to the limited literature experience in this topic and calls attention to the importance of recognizing $P I$ and its clinical significance in order to define the right conduct.
\end{abstract}

Key Words: Pneumatose Intestinal - Pneumatosis intestinalis - Pneumatosis Cystoides Intestinorum - Gas-filled colections - Intestines, cysts.

\title{
REFERÊNCIAS
}

1. Pear BL - Pneumatosis intestinalis: a review. Radiology, Apr, 1998; 207(1): 13-19.

2. Boerner RM, Friedet DB, Warshauer DM, et al - Pneumatosis intestinalis. Two case reports and a retrospective review of the literature from 1985 to 1995 . Dig-DisSci, Nov, 1996; 41(11): 2272-2285.

3. Koss LG - Abdominal gas cysts (pneumatosis cystoides intestinorum hominis): an analysis with a report of a case and a critical review of literature. Arch Pathol. 1952; 53: 523-547.

4. Heng Y, Schuffler MD, Haggitt RC, et al - Pneumatosis intestinalis: a review. Am-J-Gastroenterol. Oct, 1995; 90(10): 1747-1758.
5. Samson VE, Brown WR - Pneumatosis cystoides intestinalis in AIDS-associated cryptosporidiosis. More than an incidental finding?. J-Clin-Gastroenterol. Jun, 1996; 22(4): 311-312.

Endereço para correspondências:

Dr. Luiz Fernando Veloso.

Rua Paraisópolis, 718 / 101; Bairro Santa Teresa

31010-330 - Belo Horizonte - MG.

E-mail: luizfveloso@uol.com.br 